\title{
Campaign launched to drive routine screening for erosive tooth wear
}

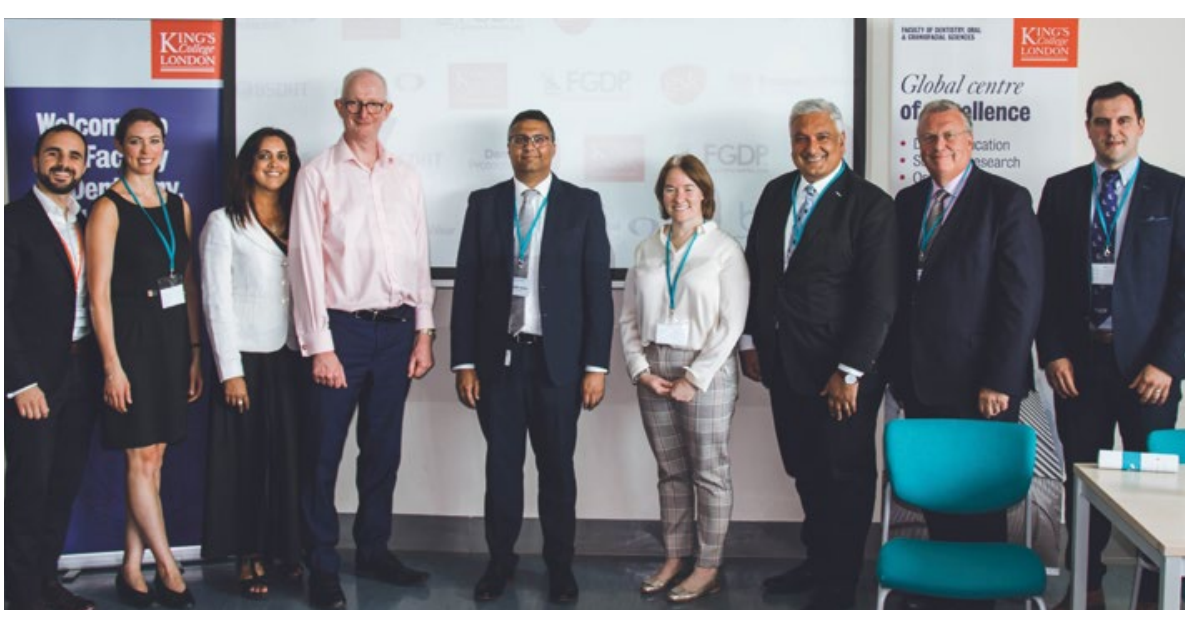

From left to right: Andrei Gutierrez, Expert Marketing Manager at GSK; Dr Saoirse O'Toole, King's College London; Dr Soha Dattani, Director Scientific \& Professional Affairs at GSK; Professor David Bartlett, King's College London; Dr Shamir Mehta, King's College London; Diane Rochford, President Elect BSDHT; Dr Raj Rattan, Dental Director at Dental Protection; Professor Nairn Wilson, Emeritus Professor of Dentistry, King's College London and Chair, Board of the College of General Dentistry; and Andrew Parashchak, Board Member, Faculty of General Dental Practitioners FGDP (UK)

\section{Leaders of the Erosive Tooth Wear}

Foundation, the Faculty of General Dental Practice (UK), the British Society of Dental Hygienists and Therapists, Dental Protection and King's College London have announced the launch of a joint campaign in partnership with GSK to drive awareness of erosive tooth wear (ETW).

Despite being the third most commonly observed oral condition, affecting up to $30 \%$ of European adults ${ }^{1}$ and not requiring a drill to fix, UK research shows that ETW is currently not routinely screened, or monitored, as part of the standard dental examination. ${ }^{2}$

These leading experts are calling on the dental profession to use the basic erosive wear examination (BEWE) alongside the basic periodontal examination (BPE) as part of every oral assessment to prompt early identification and prevention. As it follows the same sextant approach as the BPE, BEWE can be conducted at the same time, therefore requiring little additional clinical time.

Although the condition may have a slow rate of progression, the impact on aesthetics and function, as well as the financial implication of restoration, can be significant. According to a study by O'Toole et al., costs could be up to $£ 31,000$ for private treatment and the average treatment takes 21 months. ${ }^{3}$

Reports show that the rise in snacking culture and the popularity of fresh fruit and fruit juices has significantly increased the risk of developing ETW, especially when they are consumed outside of meals. Odds ratios, for example, increase nearly 12 -fold when acidic drinks are consumed on two occasions outside of meals, ${ }^{4}$ highlighting how modern diets and lifestyles have a substantial role to play.

Dr Saoirse O'Toole, Clinical Lecturer in Prosthodontics at King's College London's Faculty of Dentistry, Oral \& Craniofacial Sciences, said: 'Often when patients are referred to the hospital for treatment, it is because early signs may not have been identified and changes to behaviour have not been discussed with patients. Simple advice, such as limiting the number of acidic drinks you have and switching when you have them to mealtimes, may make a real difference in reducing patient risk.'

Comprehensive educational resources for both the clinician and patient to support this campaign are available via the Erosive Tooth Wear Foundation website: https://www. erosivetoothwear.com.

\section{References}

1. Bartlett D, Dattani $S$, Mills I et al. Monitoring erosive tooth wear: BEWE, a simple tool to protect patients and the profession. Br Dent J 2019; 226: 930-932.

2. O'Toole S, Khan M, Patel A et al. Tooth wear risk assess ment and care-planning in general dental practice. $\mathrm{Br}$ Dent J 2018; 224: 358-362.

3. O'Toole S, Pennington M, Varma S, Bartlett D W. The treatment need and associated cost of erosive tooth wear rehabilitation - a service evaluation within an NHS dental hospital. Br Dent J 2018; 224: 957-961.

4. O'Toole S, Bernabé E, Moazzez R, Bartlett D. Timing of dietary acid intake and erosive tooth wear: a case-control study. J Dent 2017; 56: 99-104.

\section{Inaugural research network meeting}

The Northern Dental Practice Based Research Network was officially launched in May 2019 at its inaugural meeting in the School of Dental Sciences, Newcastle University. The network aims to encourage, facilitate and conduct high quality research in the primary dental care setting, and to disseminate information relevant to the provision of evidence-based primary dental care.

Over 50 members attended the first meeting, comprising dentists, dental hygienists, dental therapists, dental nurses, practice managers and researchers. During the meeting members had a chance to shape the network and several volunteered to sit on a management committee. Later, members heard about completed, ongoing and future research studies conducted in the region and how they can get involved.

The event was kindly supported by a wide range of local organisations including Newcastle University, The BDA Northern Counties, The North of England

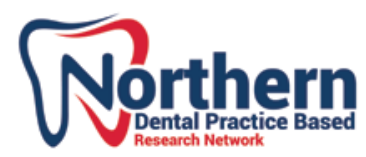

Odontological Society, North Tyne LDC and the NIHR Clinical Research Network for North East and North Cumbria.

If you are a dental professional in the North East of England and want to find out more, or join the network, visit https:// blogs.ncl.ac.uk/northerndentres or follow @NorthernDentRes on Twitter. It's a great way to find out about research opportunities in the region, big or small. 